\title{
Modern Protein Force Fields Behave Comparably in Molecular Dynamics Simulations
}

\author{
DANIEL J. PRICE, CHARLES L. BROOKS III \\ Department of Molecular Biology, TPC6, The Scripps Research Institute, 10550 North Torrey- \\ Pines Road, La Jolla, California 92037
}

Received 12 July 2001; Accepted 5 February 2002

\begin{abstract}
Several molecular dynamics simulations were performed on three proteins-bovine apo-calbindin D9K, human interleukin-4 R88Q mutant, and domain IIA of bacillus subtilis glucose permease-with each of the AMBER94, CHARMM22, and OPLS-AA force fields as implemented in CHARMM. Structural and dynamic properties such as solvent-accessible surface area, radius of gyration, deviation from their respective experimental structures, secondary structure, and backbone order parameters are obtained from each of the 2-ns simulations for the purpose of comparing the protein portions of these force fields. For one of the proteins, the interleukin- 4 mutant, two independent simulations were performed using the CHARMM22 force field to gauge the sensitivity of some of these properties to the specific trajectory. In general, the force fields tested performed remarkably similarly with differences on the order of those found for the two independent trajectories of interleukin-4 with CHARMM22. When all three proteins are considered together, no force field showed any consistent trend in variations for most of the properties monitored in the study.
\end{abstract}

(C) 2002 Wiley Periodicals, Inc. J Comput Chem 23: 1045-1057, 2002

Key words: force fields; Amber; CHARMM; OPLS-AA; protein dynamics; calbindin; interleukin-4; glucose permease

\section{Introduction}

The scope of chemical problems presently being approached with computation are of such a size and complexity that the use of potential energy functions rooted in classical physics rather than quantum mechanics is routine (here and elsewhere, the combination of the classical equations and their associated parameters is termed a force field). In particular, the broad range of computationally demanding biological problems that has been accessible through the many variants of molecular dynamics (MD) and Monte Carlo (MC) simulations in concert with modern force fields for biological macromolecules has been impressive; for example, the studies of protein motion as it relates to enzymatic catalysis, ${ }^{1}$ nucleic acid structure, and stability, ${ }^{2}$ affinity and selectivity in small molecule-protein binding, ${ }^{3}$ and the pathways of protein folding and unfolding ${ }^{4}$ have seen significant advances as a direct result of these approaches. Toward these ends, several labs have developed force fields for use in all-atom protein simulation, ${ }^{5-9}$ where validation of the force field $s$ form and parameterization against available experimental measurements and high-level $a b$ initio calculations is critical. Proteins, however, pose a particularly difficult parameterization problem for several reasons, the foremost of which is the scarcity of quantitative experimental, thermodynamic data to evaluate a parameterization's performance and isolate problematic parameters. For this reason, the development of a protein force field typically requires parameterization of small molecules that contain constituent functionality. Although combining atomic parameters from representative functional groups to construct small, polyfunctional molecules has been effective, ${ }^{10,11}$ it is difficult to assess the extent to which error accumulates in such a process for macromolecules. In addition, it should be pointed out that, unlike small molecules in solution, solvated proteins contain a variety of dielectric environments in which the force field must perform equally well.

With few exceptions, there is a consensus on the functional form of contemporary, all-atom, classical force fields. In the following, we have chosen to examine three of the most commonly used force fields for protein simulation: AMBER $94,{ }^{5}$ CHARMM $22,{ }^{8}$ and OPLS-AA ${ }^{7}$ (heretofore referred to as AMBER, CHARMM, and OPLS, respectively). A typical classical potential energy function may have the form:

Correspondence to: C. L. Brooks III; e-mail: brooks@scripps.edu Contract/grant sponsor: the National Institutes of Health; contract/grant numbers: F32 AI49673, GM 56879, GM 37554, RR 12255 


$$
\begin{array}{r}
E=\sum_{\text {bonds }} k_{b}\left(b-b_{0}\right)^{2}+\sum_{\text {angles }} k_{\theta}\left(\theta-\theta_{0}\right)^{2}+\sum_{\text {dihedrals }} V_{n}[1+\cos (n \phi \\
-\delta)]+\sum_{\text {impropers }} k_{\varphi}\left(\varphi-\varphi_{0}\right)^{2}+\sum_{U B} k_{U B}\left(U B-U B_{0}\right)^{2} \\
+\sum_{\text {nonbonded }}\left\{\varepsilon_{i j}\left[\left(\frac{R_{\text {min }}}{r_{i j}}\right)^{12}-2\left(\frac{R_{\text {min }}}{r_{i j}}\right)^{6}\right]+\frac{q_{i} q_{j}}{\varepsilon_{0} r_{i j}}\right\}
\end{array}
$$

Bonds, angles, Urey-Bradley 1-3 distances, and improper torsions are treated harmonically where $b-b_{0}, \theta-\theta_{0}, \mathrm{UB}-\mathrm{UB}_{0}$, and $\varphi-\varphi_{0}$ are the deviations from their respective equilibrium values, and $k_{b}, k_{\theta}, k_{\mathrm{UB}}$, and $k_{\varphi}$ are the force constants. Nonbonded interactions involve Coulombic and Lennard-Jones interactions for each atomic pair separated by at least three bonds, where the interaction energy of 1,4 pairs is often scaled. $R_{\min }$ and $\varepsilon_{i j}$ are the distance at which the minimum in the Lennard-Jones curve occurs and the energy at that point, respectively; $q_{i}$ and $q_{j}$ are atomic partial charges, and $\varepsilon_{0}$ is the dielectric constant. Many-body effects are typically only included in an average sense by appropriate parameterization of the pairwise nonbonded interactions. A Fourier series truncated after three or four terms supplements the torsional energetics described by the scaled nonbonded interactions.

Of course, there are some subtleties in the form of this equation from one force field to the next. For example, OPLS and AMBER use a $\mathrm{V}_{2}$-Fourier term to describe improper dihedrals

$$
E_{\text {improper }}=\frac{V_{2}}{2}[1-\cos 2 \varphi]
$$

and omit Urey-Bradley 1-3 interactions, whereas CHARMM uses the expression above without modification [eq. (1)]. In addition, OPLS uses a geometric combining rule to derive $R_{\text {min }}$ from its constituent atomic parameters

$$
R_{\min }=\sqrt{R_{\min , i} R_{\min , j}}
$$

whereas AMBER and CHARMM combine the atomic parameters arithmetically $\left(R_{\min }=1 / 2\left[\mathrm{R}_{\min , i}+R_{\min , j}\right]\right)$. For reference, $R_{\min , i}$ is related to the often-used, hard-shell radius, $\sigma_{i}$, by a constant $\left(R_{\min , i}=\sigma_{i} * 2^{1 / 6}\right)$.

However, the largest difference is clearly in the philosophies for optimization of the nonbonded parameters, where alternative parameterization procedures have been chosen in separate efforts to balance accuracy, transferability, and ease of generating new parameters for functionality beyond the scope of the original parameterization. Partial charges and Lennard-Jones parameters in OPLS are assigned empirically from iterative pure liquid and dilute solution simulations aimed at reproducing bulk condensedphase properties like densities, heats of vaporization, and relative free energies of hydration of small, organic molecules, though reproduction of $a b$ initio geometries and hydrogen-bond strengths have guided initial parameter estimates. ${ }^{7,12}$ The AMBER force field uses partial charges derived from restrained electrostatic potential (RESP) fits to ab initio charge distributions obtained at the HF/6-31G* level, ${ }^{5}$ while CHARMM was largely fit to scaled ab initio interaction energies, also at the $\mathrm{HF} / 6-31 \mathrm{G}^{*}$ level ${ }^{8}$ Charge distributions from $\mathrm{HF} / 6-31 \mathrm{G}^{*}$ calculations are generally too polar for the gas phase, ${ }^{13}$ a property that is exploited by all three procedures for nonbonded- and torsional-parameter ${ }^{14}$ optimization for incorporating the effects of polarization expected from transfer to solution. Of course, there may be a convergence of these strategies in the use of the HF/6-31G* basis and in that validation of the more automated of these procedures often require manual adjustments to fit to the same or similar experimental condensedphase data. ${ }^{5,8}$

In light of this point, perhaps a more immediate question one can ask is whether the protein portion of these force fields have arrived at a consensus for the properties or behavior of proteins, despite their different parameterization strategies. To address this issue, this study focuses on a comparative analysis of properties such as solvent-accessible surface area (SASA), radius of gyration $\left(r_{\text {gyr }}\right)$, location and geometry of secondary structure, order parameters, and atomic displacements, all computed from 2-ns molecular dynamics simulations of a small set of representative properties. These are valuable properties for comparison as they are frequently reported in the literature; for example, changes in SASA are often related to changes in entropy, and $r_{\mathrm{gyr}}$ and secondary structure are common coordinates for the progress of protein folding. ${ }^{15,16}$ Furthermore, stability of these properties during the course of multinanosecond simulations has previously aided in force field design, as was the case in an MD simulation of lysozyme using GROMOS87, where SASA and $r_{\mathrm{gyr}}$ values that increased throughout an 1.1-ns simulation aided in identifying several improvements to the force field. ${ }^{17}$ Although accuracy with respect to experiment may be difficult to gauge, identifying features of these force fields, for example if there are tendencies to produce structures that are more compact, more flexible, or have a higher propensity for certain secondary structural elements, may be useful in the design or interpretation of future computational projects. Unfortunately, many properties fluctuate on a time scale much larger than the average simulation; care must be taken that the convergence of the properties for a given simulation length be addressed.

As the goals of these simulations are in comparing the properties of several proteins using modern protein force fields, it should be noted that there has been some previous efforts in assessing the effectiveness of force fields on peptides. In particular, a comparison of gas-phase energetics of peptides to high-level ab initio data found that OPLS-AA and MMFF ${ }^{6}$ did exceptionally well at finding stable conformations near the $a b$ initio minima and with similar relative energies. ${ }^{18}$ However, electrostatics tend to dominate the gas-phase energy landscape; consequently, comparisons made in the gas phase often include conformations that are not heavily populated in solution (e.g., the $\mathrm{C} 7_{\mathrm{eq}}$ or $\alpha_{\mathrm{L}}$ conformers of a dipeptide) and vice versa (e.g., $\alpha_{\mathrm{R}}$ has no local minimum at the $\mathrm{HF} / 6-31+\mathrm{G}^{*}$ level in alanine dipeptide ${ }^{19}$ ). MacKerrell and coworkers point out that CHARMM22 does well in this comparison if one such outlier $\left(\alpha_{\mathrm{L}}\right)$ is discarded. ${ }^{8}$ It can be expected that balancing the reproduction of gas- and solution-phase properties for peptides is useful in accurately modeling interactions in the range of dielectric environments that occur in proteins, although to what extent gas-phase vs. solution-phase properties should be emphasized in the parameterization process has not been rigor- 


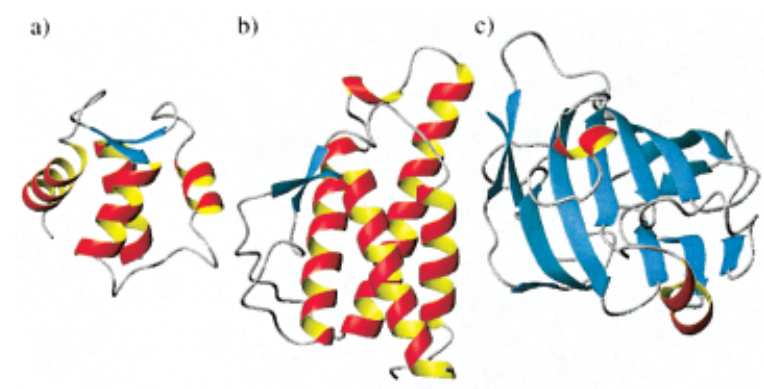

Figure 1. Model 1 from the ensemble of solution structures for calbindin (a), and the crystal structures of interleukin-4 R88Q (b), and domain IIA of glucose permease (c); $\alpha$-helices are shown in red/ yellow and $\beta$-sheets are aqua. Graphics prepared with VMD. ${ }^{75}$

ously established. In many small molecule examples, the appropriate changes in the conformational energetics are seen upon transfer from gas phase to solution with appropriate parameterization. $^{10,20}$

Three proteins that comprise a cross-section of secondary structure were chosen for simulation. Small ( $<165$ residues), singledomain proteins are preferable as they permit longer simulation times that will, in principle, minimize the bias of the initial structures, and preclude interdomain motions that would be expected to occur on a much larger time scale than the current simulations. Bovine apo-calbindin $\mathrm{D} 9 \mathrm{~K}$ is a 76-residue, righthanded, 4-helix bundle with two small $\beta$-strands, and is involved in calcium uptake from the stomach. A solution structure is available for the P43G mutant (Fig. 1a), constructed to eliminate isomerization of the peptide bond at that position (PDB code: $1 \mathrm{clb}){ }^{21}$ the mutant is simulated here for consistency. There is considerable mobility in loop regions of the apo-protein according to the NMR ensemble; the first and third loops correspond to the two unoccupied calcium-binding sites. Multiple experimental and computational efforts have been directed towards observing the loss of mobility upon calcium binding, and the interested reader is directed to the following references. ${ }^{21-23}$ Simulations here were not performed with bound ions or ligands, as we wished to focus completely on the protein portion of the force fields. The human interleukin-4 R88Q mutant (heretofore labeled IL4) is also a 4-helix bundle with two small $\beta$-strands, but the bundle is lefthanded and contains two peculiar overhand connections that may be representative of the superfamily of cytokines to which it belongs (Fig. 1b). ${ }^{24}$ The crystal structure (PDB code: 1hij) indicates that the loss-of-activity mutation does not involve significant structural changes from the wild-type protein. ${ }^{25}$ It has three disulfide bonds that are expected to limit mobility considerably; in fact, using multiple conformers in the refinement of the wild-type protein is not supported by the NMR data. ${ }^{26}$ Finally, domain IIA of bacterial glucose permease (GPIIA) is predominantly a $\beta$-barrel, or jelly-roll, topology (Fig. 1c), involved in a phosphate transfer event associated with a carbohydrate transport system unique to bacteria. (PDB entry: 1gpr). Experimental order parameters for each of these proteins (for wild-type interleukin-4) ${ }^{27-29}$ are available at the Indiana Dynamics Database (http://www.indiana.edu/ $\operatorname{idd} /) .{ }^{30}$

\section{Method}

The appropriate modifications to the energy function were implemented in the CHARMM simulation software ${ }^{31}$ to permit use of all three force fields (addition of geometric combining rule for calculating $R_{\min }$ and $\mathrm{V}_{2}$-Fourier term for improper dihedrals). The topology and parameter files that constitute the input for protein simulation in CHARMM were constructed for OPLS; the equivalent files for AMBER were previously prepared, ${ }^{32}$ and were used with only a minor amendment to the topological entry for disulfide bonds. All of the files for these three force fields are available at the URL, http://www.scripps.edu/brooks/charmm_docs/charmm. html. Gas-phase single point calculations were performed with the CHARMM simulation package and with the native software s3,34 $^{33}$ on a variety of peptides to confirm correct implementation of each force field. Energies were identical to those obtained in the force field s native program to within the limit of precision of the coordinates as they were entered in their respective input files, after correction for the different conversions from fractional electron charge units (data not shown).

a)

b)

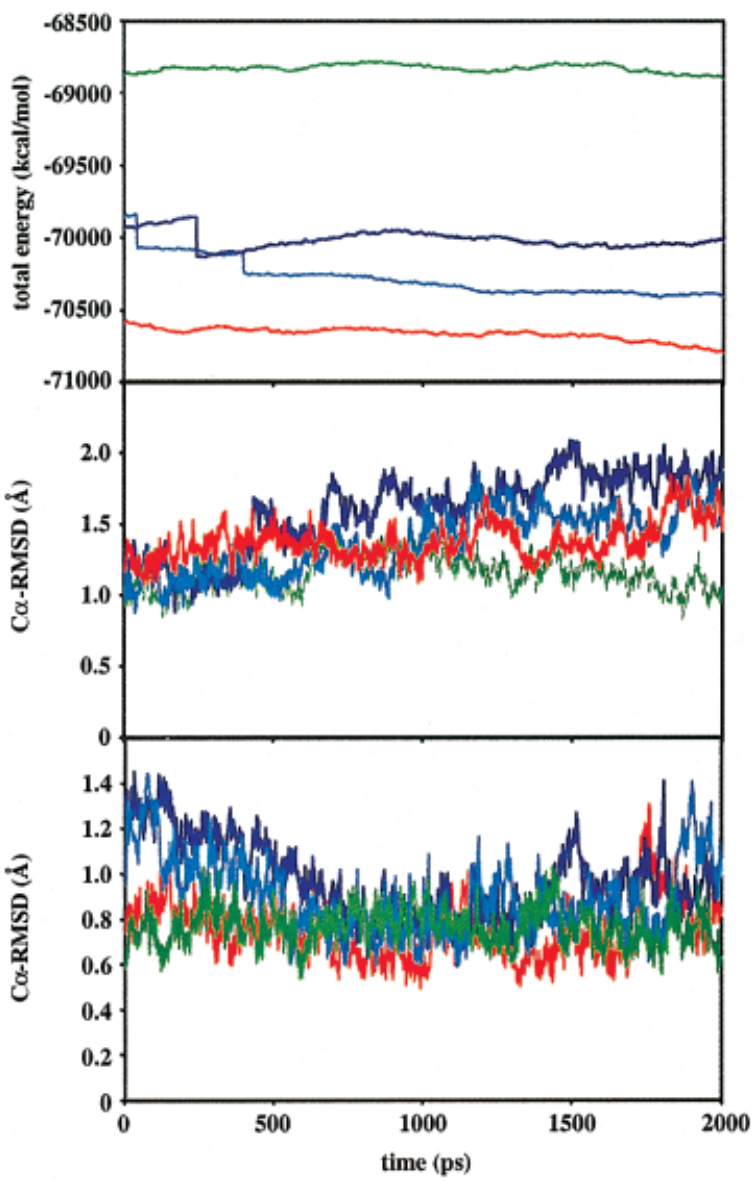

Figure 2. The total energy (a), $\mathrm{C}_{\alpha}-\mathrm{RMSD}$ to experiment (b), and $\mathrm{C}_{\alpha}$-RMSD to a simulation's own average structure (c) as a function of simulation length for IL4 with AMBER (green), CHARMM (two trajectories in aqua and blue), and OPLS (red). 
For each protein, experimentally determined coordinates were downloaded from the PDB (entries 1clb, 1hij, 1gpr) and used as starting coordinates; in the case of $1 \mathrm{clb}$, the first of the $33 \mathrm{NMR}$ models was used as it has the minimum number of experimental constraint violations. ${ }^{21}$ Missing hydrogens were added using the HBUILD utility in the CHARMM software. Histidines were automatically assigned to their $\delta$-tautomer, with the exception of His 24 and His68 of GPIIA, which were assigned to be the protonated- and $\varepsilon$-tautomers by inspection, respectively. The assignments of His83 and His68 of GPIIA are consistent with the proposed model for phosphate transfer. ${ }^{35,36}$ The simulations of GPIIA did not include the first three residues from the $\mathrm{N}$-terminus, as they were unresolved in the crystal structure. Each protein was subjected to three cycles of conjugate-gradient minimization of 500 steps with successively smaller force constants restraining the $\alpha$-carbons to the experimental structure $\left(k_{\mathrm{C} \alpha}=30,20,10 \mathrm{kcal} /\right.$ $\mathrm{mol} \approx$ ) using the CHARMM22 force field. A truncated octahedron of TIP3P water molecules ${ }^{37}$ or a modified version thereof ${ }^{38}$ was placed around the protein such that the protein was solvated with at least nine to the boundary, which translates to 3819 water molecules for calbindin, 8541 for IL4, and 5805 for GPIIA. Each periodic system was relaxed without restraints using 100 steps of steepest-descent minimization to ease poor solvent contacts at the edges of the octahedron. All simulations for a given protein were started from identical coordinates, with an equilibration period of 100 ps to allow each protein to relax under the given force field. Two simulations of IL4 were performed using the CHARMM22 force field; the initial conditions of the simulations differed only by the assigned atomic velocities.

The simulation protocol was chosen to be representative of those that are commonly used for molecular dynamics simulations in the literature. Specifically, a leapfrog integrator ${ }^{39,40}$ was used with a 2 -fs time step. Bonds to hydrogens and the internal geometry of water molecules were held rigid with the SHAKE algorithm. ${ }^{41}$ Long-range electrostatic interactions were modeled with the particle-mesh Ewald method ${ }^{42}$ using a cutoff of 8.5, $\kappa$ of 0.32 , and a grid spacing near 1 (the exact number of grid points in each dimension was 60 for calbindin, 72 for IL4, and 64 for GPIIA). The temperature was held near $298 \mathrm{~K}$ by reassignment of velocities to a Maxwell-Boltzmann distribution upon deviation from the target temperature by $5 \mathrm{~K}$. Constant pressure was obtained using the extended-system procedure of Feller and coworkers. ${ }^{43}$ For the largest system, IL4, each 2-ns simulation required $98 \mathrm{~h}$ on 16-400 MHz R12000 processors.

All analyses were performed with the CHARMM simulation package, and involve averaging properties over structures saved every 1000 time steps ( 2 ps), unless otherwise noted. The SASA calculation is analytical and uses a probe radius of 1.4 and the Lennard-Jones hard-shell radii $\left(\sigma_{i}\right)$ for each atom to define the surface. The radius of gyration $\left(r_{\text {gyr }}\right)$ is calculated with a mass weighting. All root-mean-square distances reported here are for $\alpha$-carbons only, and are denoted as $\mathrm{C}_{\alpha}-\mathrm{RMSD}$. For the purpose of identifying residues involved in secondary structure, the following definitions were used. Residues in $\alpha$-helices were assigned by visual inspection as having $\mathrm{NH}_{\mathrm{i}} \ldots \mathrm{OC}_{\mathrm{i}-4}$ hydrogen bonds (lengths less than $2.8 \AA$ ) for a sustained period with corresponding $\phi, \psi$ angles in the correct quadrant of the Ramachandran plot. Residues that were consistently in an incorrect quadrant of $\phi, \psi$ space were excluded, which affected some helix-initiating and helix-terminating residues that often stray significantly from conventional helical conformations despite having an $i \rightarrow i+4$ hydrogen bond. ${ }^{44}$ Furthermore, residues that only transiently satisfy these conditions were not included in averaging of secondary structure properties. A $\beta$-strand was defined by having multiple, sequential residues that satisfy one of two conditions: (1) having two intrastrand backbone hydrogen bonds, that is, $\mathrm{CO}_{i} \ldots N_{j}$ and $\mathrm{HN}_{i} \ldots \mathrm{CO}_{j}^{45}$ or 2) being within a $90^{\circ}$ radius of the $\phi, \psi$ combination of $-129^{\circ}$, $123^{\circ},{ }^{46}$ and in a strand with a residue that satisfies condition 1. This definition allows for small kinks and bulges in the $\beta$-sheets, as well as bonded strands of different lengths, but not isolated extended structure. Reported uncertainties have been corrected for correlation between frames by multiplying by their relaxation times. ${ }^{47}$ The uncertainty associated with the relaxation time increases with its magnitude, however, as the autocorrelation function is constructed from fewer points at longer lag times; consequently, the maximum uncertainty for a given property from all simulations is used as an estimate for each individual simulation in an effort to compensate for this undetermined uncertainty in the relaxation times.

Gas-phase calculations on model systems were performed in the CHARMM simulation package. Implicit solvent calculations using the generalized Born (GB) model ${ }^{48,49}$ were performed in the native software to ensure the appropriate functional form and parameterization of the GB equation was used for each force field. ${ }^{49-51}$

\section{Results}

\section{Overall Properties}

The total energies and temperature are stable throughout all simulations (averages are reported in Table 1; a time course of total energies for IL4 is shown in Fig. 2a). Of course, one would not expect to be able to compare the absolute energies, as they are specific to the parameterization and the form of the potential energy function; however, the standard deviations of the total energy suggest a similar value for the system's heat capacity. A quantitative comparison of these values would clearly benefit from longer simulations as the standard deviations are biased by the number of temperature reassignments that occurred during the averaging period; the AMBER simulations did not have such a reassignment, and consequently, have the smallest standard deviations. At most, two temperature reassignments occurred during the CHARMM and OPLS simulations and always towards their beginning, suggesting that thermalization continued to some extent on a longer time scale than the initial 100-ps equilibration. RMSD to their respective experimental structures was also stable; the absence of a continuous drift away from the starting conformation throughout the simulations indicates that a structure or a collection of structures near the experimental geometry represents a local minimum in each force field. No force field consistently sampled structures closer to the experimental geometry for all proteins than the others. Figure $2 \mathrm{~b}$ shows the trajectory of the $\mathrm{C}_{\alpha}$-RMSD for each of the force fields for IL4 relative to the crystal structure. The magnitude to which two simulations may deviate given the same 
Table 1. Averaged Overall Properties (Standard Deviations in Parenthesis).

\begin{tabular}{|c|c|c|c|c|c|c|c|c|c|}
\hline & & $\begin{array}{c}\text { Total Energy } \\
(\mathrm{kcal} / \mathrm{mol})\end{array}$ & $\mathrm{T}\left({ }^{\circ} \mathrm{C}\right)$ & $\begin{array}{l}C_{\alpha} \operatorname{RMSD}(\AA) \\
\text { to Experiment }\end{array}$ & $\begin{array}{c}C_{\alpha} \text {-RMSD }(\AA) \\
\text { to Average }\end{array}$ & $r_{\mathrm{gyr}}(\AA)$ & $\begin{array}{l}\quad r_{\mathrm{gyr}}(\AA) \\
\text { Experiment }\end{array}$ & SASA $\left(\AA^{2}\right)$ & $\begin{array}{l}\text { SASA }\left(\AA^{2}\right) \\
\text { Experiment }^{\mathrm{a}}\end{array}$ \\
\hline \multicolumn{10}{|c|}{ Calbindin } \\
\hline & AMBER & $-31085(39)$ & $298(2)$ & $3.02(0.20)$ & $1.05(0.16)$ & $11.70(0.07)$ & $11.42^{\mathrm{b}}$ & $5225(70)$ & $4763^{b}$ \\
\hline & CHARMM & $-31406(68)$ & 299 (2) & $2.76(0.13)$ & $1.01(0.18)$ & $11.88(0.08)$ & & 5253 (99) & $4761^{\mathrm{b}}$ \\
\hline & OPLS & $-33104(76)$ & $299(2)$ & $2.63(0.17)$ & $0.98(0.21)$ & $11.70(0.07)$ & & 5017 (97) & $4778^{\mathrm{b}}$ \\
\hline \multicolumn{10}{|l|}{ IL4 } \\
\hline & AMBER & $-68836(26)$ & $300(1)$ & $1.14(0.12)$ & $0.76(0.09)$ & $14.76(0.07)$ & 14.55 & 7763 (168) & 7084 \\
\hline & CHARMM 1 & $-70287(124)$ & $299(2)$ & $1.59(0.27)$ & $0.97(0.17)$ & $15.00(0.13)$ & & $8050(223)$ & 7027 \\
\hline & CHARMM 2 & $-70019(60)$ & $300(2)$ & $1.36(0.13)$ & $0.89(0.17)$ & $14.82(0.06)$ & & 7814 (118) & 7027 \\
\hline & OPLS & $-70670(43)$ & $300(1)$ & $1.37(0.13)$ & $0.75(0.13)$ & $14.79(0.06)$ & & 7661 (104) & 7077 \\
\hline \multicolumn{10}{|l|}{ GPIIA } \\
\hline & AMBER & $-47239(32)$ & $300(2)$ & $0.94(0.10)$ & $0.62(0.07)$ & $14.71(0.05)$ & 14.61 & 7724 (103) & 7503 \\
\hline & CHARMM & $-47130(100)$ & $300(2)$ & $1.20(0.15)$ & $0.74(0.11)$ & $14.78(0.06)$ & & 7799 (96) & 7458 \\
\hline & OPLS & $-50356(55)$ & $300(2)$ & $1.25(0.26)$ & $0.84(0.12)$ & $14.81(0.08)$ & & 7879 (153) & 7496 \\
\hline
\end{tabular}

${ }^{\text {a } H y d r o g e n s ~ w e r e ~ b u i l t ~ o n t o ~ t h e ~ c r y s t a l ~ s t r u c t u r e s ~ f o r ~ I L 4 ~ a n d ~ G P I I A ~(h y d r o g e n s ~ w e r e ~ a v a i l a b l e ~ f o r ~ a l l ~ c a l b i n d i n ~ N M R ~}$ structures), and subsequent SASA calculations were performed using the Lennard-Jones parameters of each force field. bSASA and $r_{\text {gyr }}$ calculations were performed on all 33 models of the calbindin NMR ensemble and averaged.

starting position but different starting velocities can be appreciated with the two simulations of IL4 using CHARMM (the average $\mathrm{C}_{\alpha}$-RMSD from the crystal structure changes by 0.23 - ca. $15 \%$ ). It is interesting to note that of the two CHARMM22 simulations of IL4, the trajectory that remains closer to the experimental structure is also lower in total energy. Regardless, comparing the range of average $\mathrm{C}_{\alpha}$-RMSD for each force field falls near this difference. The slightly cupped shape of the $\mathrm{C}_{\alpha}$-RMSD from the average structure in some simulations suggests that these simulations are still exploring the available conformational space within the vicinity of the experimental geometry (Fig. 2c). Table 2 contains a matrix of cross- $C_{\alpha}-$ RMSD values for the average structure from each trajectory to each other and their respective experimental structures. On the whole, the average structures are as near to each other as they are to the experimental structure. The one exception is in calbindin, where the average structures from the AMBER and CHARMM simulations are closer to each other than they are to the average of the NMR ensemble.

No crystal structure is available for apo-calbindin; $\mathrm{C}_{\alpha}-\mathrm{RMSD}$ to the average NMR structure is reported in Table 1. As an average structure may not be physically realistic (e.g., nonphysical bond lengths), the $\mathrm{C}_{\alpha}$-RMSD to all 33 NMR models was also calculated. The simulations were started from model 1 ; consequently, it is not unexpected that all calbindin simulations remained closest to model 1 ( $\mathrm{C}_{\alpha}$-RMSD is ca. $2.0 \AA$ in all cases). The average $\mathrm{C}_{\alpha}-\mathrm{RMSD}$ to the remaining NMR models ranged between $2.2 \AA$ and $3.3 \AA$ for all force fields, with models $2,4,5,6,24,25$, and 26 being on the closer end of the gamut. Not surprisingly, these seven models are

Table 2. $\mathrm{C}_{\alpha}$-RMSD Between Average Structures from Each Trajectory and Their Respective Experimental Structures.

\begin{tabular}{lccccr}
\hline Calbindin & AMBER & CHARMM & OPLS & NMR average & \\
AMBER & 0.00 & 1.28 & 2.49 & 2.82 & \\
CHARMM & & 0.00 & 2.23 & 2.56 & \\
OPLS & & & 0.00 & 2.45 & \\
NMR & & & & 0.00 & \\
average & & & & OPLS & 0.86 \\
IL4 & AMBER & CHARMM1 & CHARMM2 & 0.95 & 1.28 \\
AMBER & 0.00 & 1.12 & 0.90 & 1.48 & 1.04 \\
CHARMM1 & & 0.00 & 1.13 & 0.25 & 0.00 \\
CHARMM2 & & & 0.00 & 0.00 & \\
OPLS & & & & & \\
Crystal & & & OPLS & 0.71 & \\
GP-IIA & AMBER & CHARMM & 0.84 & 0.96 & \\
AMBER & 0.00 & 0.92 & 0.87 & 0.96 & \\
CHARMM & & 0.00 & 0.00 & 0.00 & \\
OPLS & & & & & \\
Crystal & & & & & \\
\end{tabular}


Table 3. Location of Secondary Structure and Average Hydrogen Bond Lengths (Standard Deviation in Parentheses).

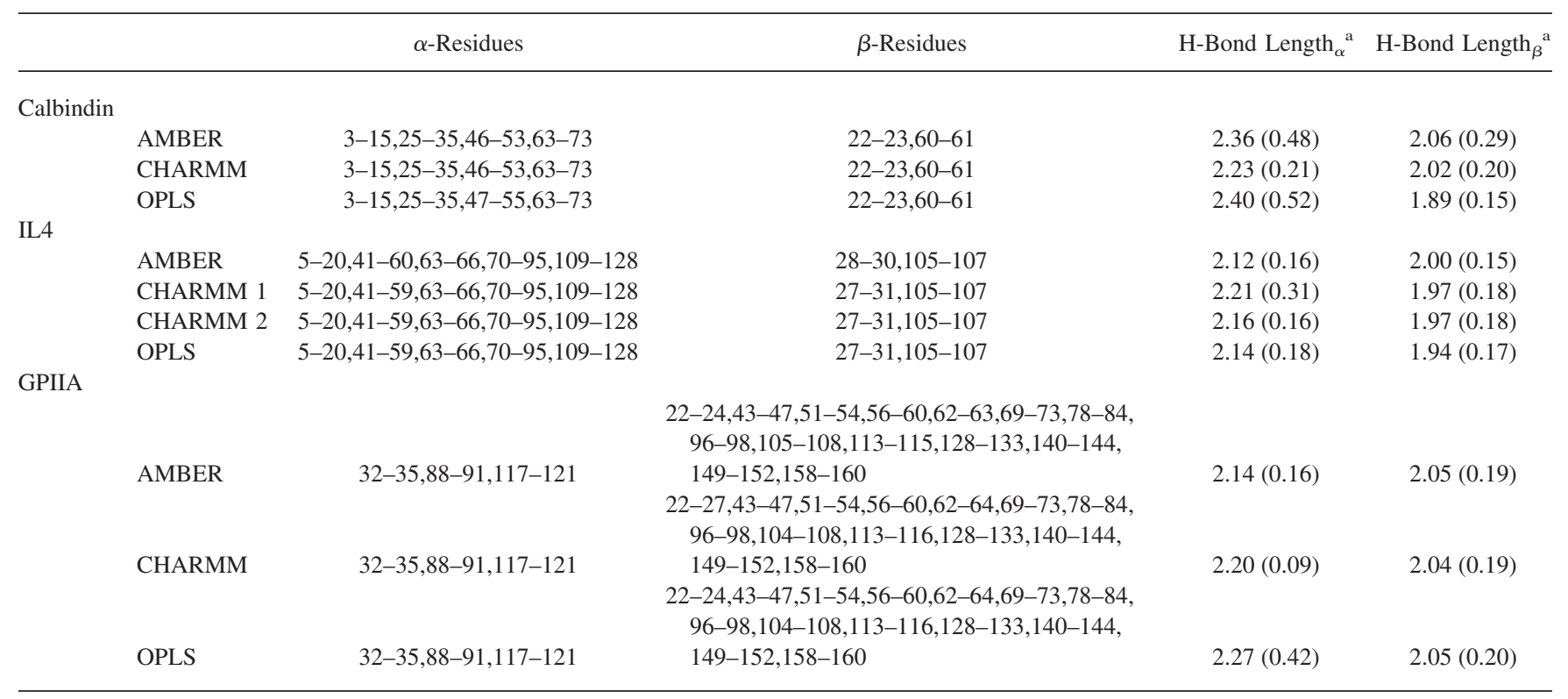

${ }^{a}$ Averages of secondary structure included only those residues that were consistently involved in $\alpha$ - or $\beta$-structure throughout all simulations: for calbindin, residues 3-12,22-23,25-35,60-61,63-73; for IL4, residues 6-18,28-30,4158,70-94,105-107,109-126; and for GPIIA, residues 22-24,32-35,44-46,51-53,59-60,62-63,69-73,78-84, 88-91,96-98,105-106,113-115,117-121,131-133,143-144,158-160.

those showing the smallest $\mathrm{C}_{\alpha}$-RMSD to model $1\left(\mathrm{C}_{\alpha}\right.$-RMSD is 1.4-1.7, compared to the average $\mathrm{C}_{\alpha}$-RMSD from model 1 of 1.8 $\AA$ ). Interestingly, the range of $\mathrm{C}_{\alpha}$-RMSD of the simulation structures to the experimental ones for all force fields here is substantially larger than what is seen in the simulations of the other proteins and larger than the average $\mathrm{C}_{\alpha}$-RMSD between the NMR models $(2.0 \AA)$, and suggests that calbindin is less rigid than the other proteins and that the collection of minimized NMR models may not completely demonstrate that motion.

SASA and $r_{\text {gyr }}$ were monitored as gross measures of compactness; average values are listed in Table 1. Again, it is beneficial to consider the difference between the two trajectories of IL4 with CHARMM when comparing between force fields. In fact, the SASA from the first IL4 simulation with CHARMM is two standard deviations greater than the second $\left(236 \AA^{2}\right)$. The range of average SASA in the calbindin and GPIIA simulations are less than that difference. There is an intuitive correlation between the compactness as measured by SASA and $r_{\text {gyr }}$ and the size of the average atomic fluctuations of the protein that will be discussed more below. No force field produced consistently the most or least compact structure, although AMBER was consistently more compact than CHARMM by 25 to $287 \AA^{2}$, and correspondingly showed smaller average atomic fluctuations. All three force fields agree that the $r_{\text {gyr }}$, and correspondingly the SASA, is slightly larger in simulations at finite temperature and in aqueous solution than those determined for the refined experimental structures (Table 1). The SASA of the experimental structures was calculated using the Lennard-Jones parameters of each force field and show some variation $\left(2-57 \AA^{2}\right.$ ), where CHARMM produced areas slightly, though consistently, smaller than the other force fields, and may suggest even closer agreement in the compactness predicted by AMBER and CHARMM than originally indicated. The minimum SASA values (data not shown) did not correlate with the average SASA values, indicating that a smaller average SASA does not necessarily measure a force field $s$ capacity to find compact structures.

\section{Secondary Structure and Backbone Conformation}

Automated secondary structure assignment uses varied definitions in the literature including hydrogen-bond strength using a simplied potential, hydrogen-bond geometries, and/or $\phi, \psi$ combinations, and most require some minimum repetition of these elementary units. ${ }^{45,52}$ In addition to semantics, the fact that some residues may only occupy secondary structure transiently in a simulation makes a secondary structure assignment that is consistent with that in the literature and the PDB difficult. The definitions for $\alpha$ - and $\beta$-structure used here are given in the Methods section, the results of which are reported in Table 3. Given this definition, there is strong overall agreement among the force fields, and only minor discrepancies with the $\operatorname{DSSP}^{45}$ assignment available at the PDB. In particular, the location of the $\beta$-sheets found in loop regions of both calbindin and IL 4 are shifted by one residue in all simulations here relative to the experimental assignment, and are of slightly different length. There is also some discrepancy in the location and length of helix 3 of calbindin when comparing the simulation with OPLS and the simulations with CHARMM and AMBER. 


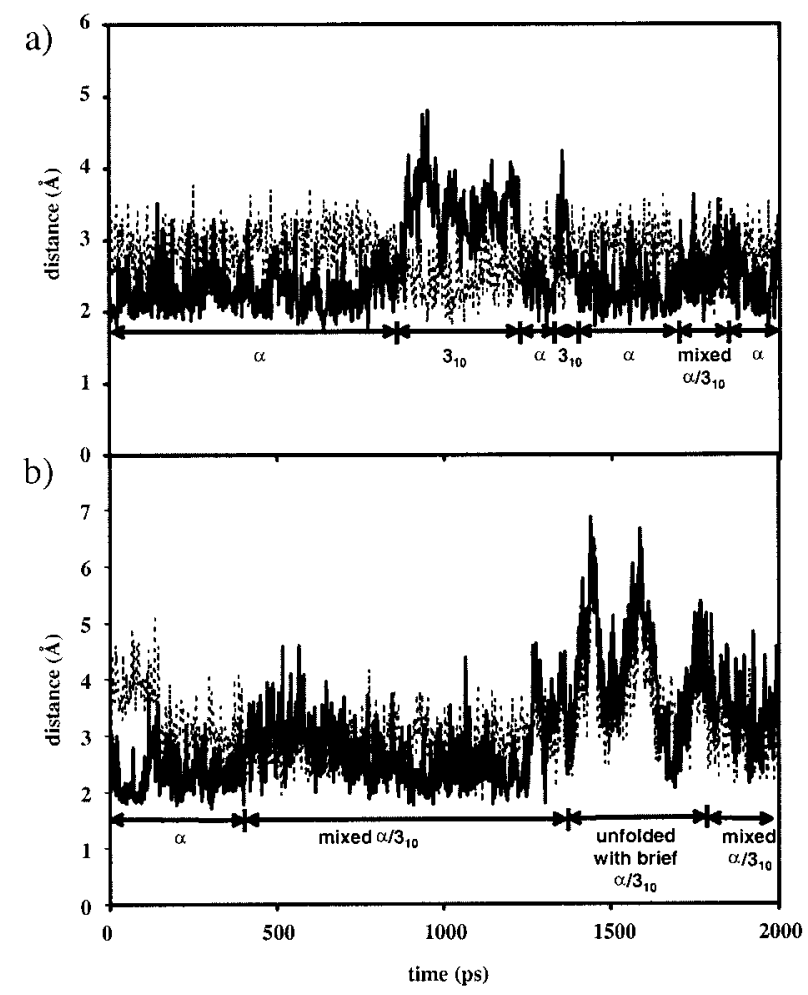

Figure 3. (a) The trajectory of $\mathrm{CO}_{26} \ldots \mathrm{HN}_{30}$ (solid) and $\mathrm{CO}_{27} \ldots \mathrm{HN}_{30}$ (dotted) from AMBER simulation of calbindin. (b) The trajectory of $\mathrm{CO}_{15} \ldots \mathrm{HN}_{19}$ (solid) and $\mathrm{C}_{16} \mathrm{O} \ldots \mathrm{HN}_{19}$ (dotted) from first CHARMM simulation of IL4.

Not included in these definitions is any indication of the duration for which a residue is involved in the secondary structure. This property is interesting, considering that a time course of hydrogenbond lengths reveal that between 95 and $100 \%$ of the helical residues in each simulation have periods where the $i \rightarrow i+3$ hydrogen bond is shorter than the $i \rightarrow i+4$ hydrogen bond, resulting in a helical conformation that resembles more closely a $3_{10}$ helix. Figure 3 a shows an example where there is a distinct transition to a $3_{10}$ helix for ca. 400 ps before returning to a conventional $\alpha$-helix. In fact, conformations with shorter $i \rightarrow i+$ 3 hydrogen bonds occur in $8-9 \%$ of the IL4 simulation snapshots and between $15 \%$ (CHARMM) and 25\% (OPLS) of the calbindin simulations, although a mixed, helical conformation that involves a bifurcated hydrogen bond to the $i+3$ and $i+4$ residues and/or facile exchange between them accounts for much of these percentages (Fig. 3b). No consistent trends of one force field visiting $3_{10}$ geometry more or less frequently are apparent when the proteins are considered together. Also, there are no obvious trends to the location and type of the few residues ( $\leq 3$ per simulation) that were consistently $\alpha$.

A trajectory of hydrogen-bond lengths or $\phi, \psi$ values also show that there are one or more residues on several of the helix ends whose secondary structure dissolves and occasionally reforms during the simulation. Residue 19 of the first IL4 simulation with CHARMM is illustrative of the helix fraying seen (Fig. 3b), where the melting of the helical conformation at $1.3 \mathrm{~ns}$ closely follows that of residue 20. For most of these local folding/refolding events, only one to two transitions between on and off configurations are seen; therefore, a potential of mean force (pmf) for this process would be poorly converged and inaccurate. However, $1-2$ ns is a reasonable time scale for this transition considering the helix propagation occurs in a model helical system on a similar time scale $(2-10 \mathrm{~ns}){ }^{53}$

To more closely examine the helical preferences, distributions of the $i \rightarrow i+4$ and $i \rightarrow i+3$ hydrogen bonds were constructed (those for IL4 are shown in Fig. 4a-d). The AMBER $i \rightarrow i+4$ profiles for both calbindin and IL4 (GPIIA has too few helical hydrogen bonds to be reliable) are slightly taller and narrower, and may reflect the $\alpha$-helical propensity that has been observed in this version of AMBER before. ${ }^{54}$ Otherwise, the distributions are very similar, and suggest a similar occupancy of helical conformations and a similar range of allowable helical motions, two properties that may be intrinsically related to the tendency for helix fraying. One proposed mechanism for helix denaturation involves passing

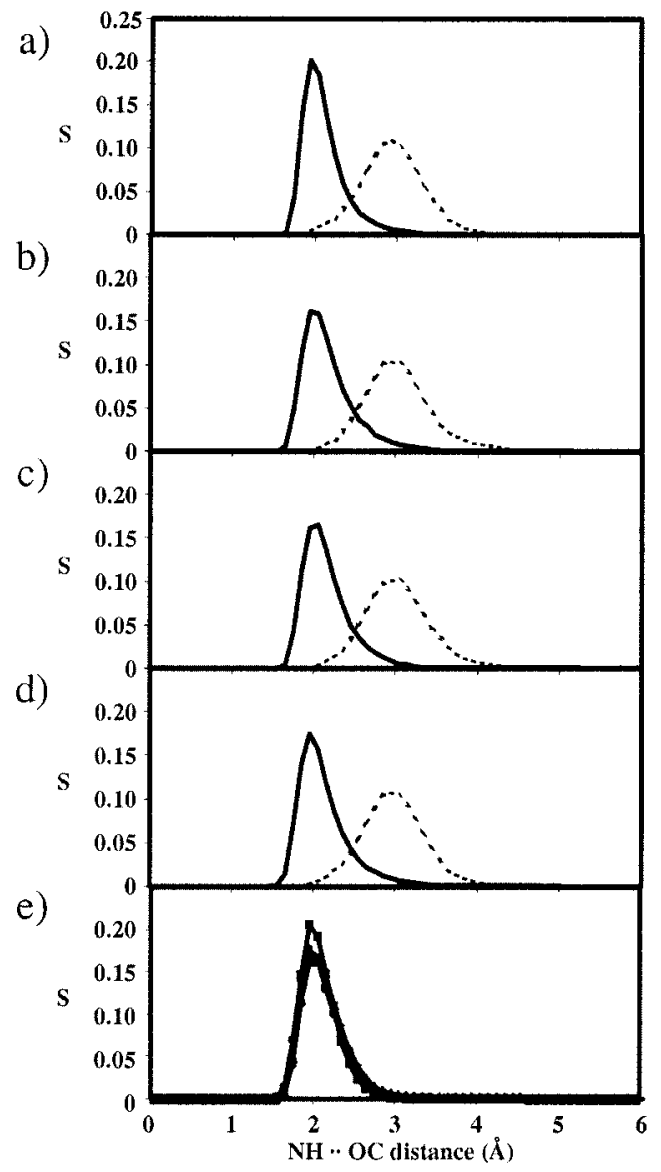

Figure 4. Hydrogen bond distributions for helices in IL4 for simulations with AMBER (a), CHARMM (b-c), and OPLS (d). Distribution of $i \rightarrow i+4$ hydrogen-bond lengths is shown as solid, $i \rightarrow i+3$ as dashed. (e) The distribution of the minimum of $i \rightarrow i+4$ and $i \rightarrow$ $i+3$ hydrogen-bond lengths for AMBER (squares), two trajectories with CHARMM (triangles and X), and OPLS (circles). 
Table 4. Hydrogen-Bond Strength in Minimized Model Systems of $N$-methyl Acetamide (NMA) and TIP3P Water.

\begin{tabular}{|c|c|c|c|c|c|c|c|c|c|}
\hline & \multirow[b]{2}{*}{$E_{\text {NMA-NMA }}$} & \multirow[b]{2}{*}{$E_{\mathrm{NMA}}$} & \multirow[b]{2}{*}{$\Delta E_{\mathrm{NMA}-\mathrm{NMA}}$} & \multicolumn{3}{|c|}{$\mathrm{CO} \ldots \mathrm{HOl}$} & \multicolumn{3}{|c|}{$\mathrm{NH} \ldots \mathrm{OH}_{2}$} \\
\hline & & & & $E_{\mathrm{NMA}-w a t e r}$ & $\Delta E_{\mathrm{NMA} \text {-water }}$ & $\Delta \Delta E_{\text {NMA-NMA } \rightarrow \text { NMA-water }}$ & $E_{\text {NMA-water }}$ & $\Delta E_{\mathrm{NMA}-\text { water }}$ & $\Delta \Delta E_{\mathrm{NMA}-\mathrm{NMA} \rightarrow \text { NMA-water }}$ \\
\hline AMBER & -70.68 & -30.84 & -9.00 & -39.62 & -8.78 & 0.22 & -36.70 & -5.86 & 3.14 \\
\hline CHARMM & -70.15 & -30.67 & -8.81 & -38.92 & -8.25 & 0.56 & -36.92 & -6.25 & 2.56 \\
\hline OPLS & -41.65 & -16.44 & -8.77 & -24.41 & -7.97 & 0.80 & -22.83 & -6.39 & 2.38 \\
\hline
\end{tabular}

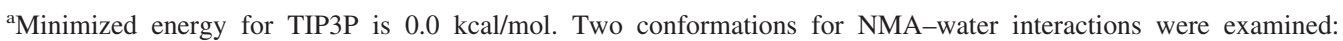
carbonyl oxygen to water hydrogen $(\mathrm{CO} \ldots \mathrm{HOH})$ and amide hydrogen to water oxygen $\left(\mathrm{NH}_{\ldots} \ldots \mathrm{OH}_{2}\right)$

through a $310^{- \text {like conformation as an intermediate. }}{ }^{55-57} \mathrm{~A}$ second mechanism involves the insertion of a water molecule into the backbone hydrogen bond, though Daggett and Levitt contend that this event is not the primary driving force behind denaturation, but secondary to internal fluctuations that force the geometry outside typical helical character. ${ }^{58}$ If this is the case, one can examine the distribution of the minimum of the $i \rightarrow i+4$ and $i \rightarrow i+3$ hydrogen-bond lengths (Fig. 4e) and compare the occupancy of conformations that have extended backbone hydrogen bonds with the assumption that these conformations have a propensity to fully denature upon the intercalation of a water molecule. The similar shapes of these composite $i \rightarrow i+4$ and $i \rightarrow i+3$ distributions argue that the more extended conformations that are a result of stretching motions in a helix occur with a similarly rare frequency in simulations with each of the force fields. In fact, no force field consistently had the largest or smallest occupancies for the region where both $i \rightarrow i+3$ and $i \rightarrow i+4$ hydrogen bond distances were greater than $3.5 \AA$, although it should be noted that this part of the distribution is poorly occupied and, therefore, the least well converged. Finally, it may be of interest that there is some consistency in the location of hydrogen bonds that experience these stretching motions. For example, the terminal hydrogen bond of helix 1 in IL4, $\mathrm{CO}_{16} \ldots \mathrm{H}_{20} \mathrm{~N}$ completely denatures at $1.3 \mathrm{~ns}$ of the first CHARMM simulation of IL4, but all of the simulations had stretching motions such that both the $\mathrm{CO}_{16} \ldots \mathrm{HN}_{20}$ and $\mathrm{C}_{17} \mathrm{O} \ldots \mathrm{HN}_{20}$ temporarily exceed 3.5 . Similarly, residue 46 at the $\mathrm{C}$-terminal end of helix 3 in calbindin was unstable in the OPLS simulations, forming the $\mathrm{CO}_{46} \ldots \mathrm{HN}_{50}$ hydrogen bond only half of the simulation and never sampling in the $\alpha$-helical quadrant of $\phi, \psi$ space. Again, the remaining simulations experienced stretched helical conformations as well. All three force fields also produced stretching motions at $\mathrm{CO}_{11} \ldots \mathrm{HN}_{15}, \mathrm{CO}_{8} \ldots \mathrm{HN}_{12}$, $\mathrm{CO}_{4} \ldots \mathrm{HN}_{8}$ calbindin; helix 1 frays at residue 15 in the CHARMM simulation, helix 1 frays at residue 8 in the AMBER simulation, and the common stretching motions for $\mathrm{CO}_{8} \ldots \mathrm{HN}_{12}$ occur near where a kink is expected in helix 1 according to the solution ensemble.

It may be expected that the similar shape of helix hydrogen bond distributions are a result of a convergence of the strength of the amide-amide hydrogen bond within these force fields. This more microscopic property can be probed with the model systems, $\mathrm{N}$-methyl acetamide (NMA) dimer and NMA-water pair. Interestingly, the energy of the gas-phase amide-amide hydrogen bond is quite similar ( $\Delta E_{\text {NMA-NMA }}$ ) between the force fields (Table 4$)$, as is the sum of the $\Delta \Delta E_{\mathrm{NMA}-\mathrm{NMA} \rightarrow \mathrm{NMA} \text {-water }}$ for the two amidewater minima (the range of both is ca. $0.2 \mathrm{kcal} / \mathrm{mol}$ ). However, the contribution from the amide $\mathrm{N}-\mathrm{H}$ and $\mathrm{C}=\mathrm{O}$ is divided differently in each force field, as evident by the individual $\Delta \Delta E$. This difference may impact the relative rates that $\mathrm{C}$-terminal and $\mathrm{N}$-terminal helix denaturation occur, though much longer simulations would be required to explore this possibility. Beachy et al. used gas-phase cis-NMA dimers to model hydrogen-bond interactions in $\beta$-sheets, and found similar association energies for CHARMM and $\operatorname{AMBER}(\Delta E=0.27 \mathrm{kcal} / \mathrm{mol}) .{ }^{18}$ Their results for the bifurcated hydrogen-bonding interaction of cis-NMA corroborates the similar magnitude of interaction energy found here for trans-NMA, although they do point out that the interpretation of these results in the context of proteins is difficult. The discrepancy between AMBER and CHARMM increases when they repeat the calculations on the larger model system of alanine dipeptide dimer and is conformationally sensitive $\left(0.84-2.4 \mathrm{kcal} / \mathrm{mol}^{59}\right)$, suggesting a significant steric interaction between the carbonyl oxygen and the $\alpha$-carbons on the neighboring backbone.

As a consequence of the residues that are intermittently involved in helices, a comparison of average hydrogen-bond lengths and $\phi, \psi$ values would be biased by the duration of the off-helix period. However, those residues that permanently subscribe to the aforementioned definitions for helical structure can be surveyed; average hydrogen-bond lengths and $\phi, \psi$ values for these residues are shown in Tables 3 and 5. Hydrogen-bond lengths for $\alpha$ - and $\beta$-structure are nearly identical for IL4 between the force fields (the first CHARMM trajectory, which experienced larger average atomic fluctuations, is slightly larger), as are the $\beta$ regions of GPIIA. The larger range of values for calbindin and the helical regions of GPIIA most likely reflect their heightened mobility, as indicated by their larger standard deviations. A recent survey of a representative set of proteins from the PDB noted that hydrogen bond lengths in $\alpha$-helices were ca. $0.2 \AA$ longer than those in $\beta$-sheets, ${ }^{60}$ a trend that is reproduced here in all simulations with all force fields.

For the core secondary structure of each protein (the $\alpha$-helices in IL4 and calbindin, and the $\beta$-barrel in GPIIA), the average $\phi, \psi$ values are very similar with differences between $0-4^{\circ}$, with the larger differences in the more mobile calbindin. Notably, there is a strong consensus among the force fields for the average $\phi, \psi$ values for the $\beta$-strands in GPIIA, despite the fact that the $\beta$-sandwich fold causes substantial deviations from $\beta$-geometries averaged over sample structures from the $\operatorname{PDB}\left(\phi_{\beta}, \psi_{\beta}=-129^{\circ}\right.$, 
Table 5. Average Dihedral Angles (Standard Deviation in Parentheses).

\begin{tabular}{|c|c|c|c|c|c|c|c|c|c|c|}
\hline & & $\phi_{\alpha}{ }^{\mathrm{a}}$ & $\psi_{\alpha}{ }^{\mathrm{a}}$ & $\phi_{\beta}{ }^{\mathrm{a}}$ & $\psi_{\beta}{ }^{\mathrm{a}}$ & $\chi_{1}$ & $\omega_{1}$ & $\left|\omega_{1}-180\right|$ & NH impr & $\mid \mathrm{NH}$ impr| \\
\hline \multicolumn{11}{|l|}{ Calbindin } \\
\hline & AMBER & $-66(11)$ & $-39(11)$ & $-116(15)$ & $136(15)$ & 223 (17) & $177.5(7.9)$ & $8.0(5.6)$ & $-0.2(5.8)$ & $5.0(3.7)$ \\
\hline & CHARMM & $-65(10)$ & $-42(10)$ & $-119(13)$ & 145 (10) & 213 (13) & $178.2(7.2)$ & $6.9(5.0)$ & $0.3(4.7)$ & $3.8(2.9)$ \\
\hline & OPLS & $-63(10)$ & $-43(9)$ & $-115(15)$ & 145 (10) & 214 (14) & $177.4(7.4)$ & $7.4(5.2)$ & $-1.1(5.7)$ & $4.9(3.6)$ \\
\hline \multicolumn{11}{|l|}{ IL4 } \\
\hline & AMBER & $-62(9)$ & $-43(9)$ & $-107(15)$ & $153(10)$ & $226(15)$ & $177.0(7.2)$ & $7.6(5.3)$ & $-0.2(5.6)$ & $4.8(3.6)$ \\
\hline & CHARMM1 & $-64(10)$ & $-42(9)$ & $-103(12)$ & $150(11)$ & 220 (13) & $178.1(7.1)$ & $7.2(5.0)$ & $0.1(4.6)$ & $3.8(2.9)$ \\
\hline & CHARMM2 & $-64(9)$ & $-42(9)$ & $-104(13)$ & 154 (11) & $220(15)$ & $177.6(7.0)$ & $7.2(5.0)$ & $0.2(4.6)$ & $3.8(2.9)$ \\
\hline & OPLS & $-62(9)$ & $-44(8)$ & $-99(12)$ & 147 (10) & 222 (12) & $177.0(6.9)$ & $7.4(5.1)$ & $-1.1(5.6)$ & $4.8(3.6)$ \\
\hline \multicolumn{11}{|l|}{ GPIIA } \\
\hline & AMBER & $-66(11)$ & $-33(11)$ & $-109(14)$ & 135 (13) & 205 (16) & $177.4(8.0)$ & $8.6(5.8)$ & $-1.1(5.9)$ & $5.3(3.8)$ \\
\hline & CHARMM & $-69(11)$ & $-35(11)$ & $-108(12)$ & 135 (10) & 209 (14) & $178.9(7.4)$ & $7.7(5.3)$ & $-0.3(4.7)$ & $4.0(3.0)$ \\
\hline & OPLS & $-67(12)$ & $-37(10)$ & $-110(13)$ & 138 (11) & 204 (12) & $177.6(7.6)$ & $8.1(5.5)$ & $-2.1(5.8)$ & $5.4(3.9)$ \\
\hline
\end{tabular}

${ }^{\text {a }}$ See Table 3 for note on which residues were used for averaging secondary structure properties.

$\left.123^{\circ 46}\right)$. The average $\phi, \psi$ values for helices from all simulations are centered on the results of a surveys of crystal structures $\left(\phi_{\alpha}\right.$, $\left.\psi_{\alpha}=-65^{\circ},-41^{\circ}\right){ }^{61}$ Deviations from ideal $\alpha$-helical geometry $\left(\phi_{\alpha}, \psi_{\alpha}=-57^{\circ},-47^{\circ}\right)^{52}$ is presumably a result of exchange with and/or partial occupancy of $3_{10}$ helical conformation. The $\phi, \psi$ values of the noncore regions of secondary structure $(\beta$-strands of IL4 and calbindin and helices of GPIIA), are averaged over only a few residues. For this reason, and the fact that these sheets and helices are located in the more mobile loop regions of their respective proteins, it is not surprising that there is a larger spread in these values, as exemplified by the two CHARMM trajectories of IL4. In considering all of the averages together, no force field produces $\phi_{\beta}, \psi_{\beta}$, or $\phi_{\alpha}$ averages that are consistently smaller or larger than the others, and although the $\psi_{\alpha}$ from the OPLS simulations is consistently more negative and has a slightly smaller standard deviation relative to the values obtained from the other force fields, the significance of this observation is dubious considering the uncertainty in the averages $\left(\mathrm{ca} .1^{\circ}\right)$.

Examination of less common secondary structure isolates some subtle differences in the force fields. In particular, analysis of the frequency and stability of $\beta$-turns and $3_{10}$ helices in GPIIA suggests an increased stability for $\mathrm{NH}_{\mathrm{i}} \ldots \mathrm{CO}_{\mathrm{i}-3,4}$ hydrogen bonds outside of the context of $\alpha$-helices in AMBER. In the AMBER simulation, $15 \beta$-turns and 1 short $3_{10}$ helix (residues 87 to 92 ) are found (where a turn is defined here as having an average backbone hydrogen-bond lengths of less than $2.8 \AA$ ), vs. only 11 and 8 turns in the OPLS and CHARMM simulations, respectively, and no $3_{10}$ helices in either. Furthermore, the average hydrogen-bond length for these turns is smaller in AMBER and with smaller fluctuations (2.28 $\AA, \sigma=0.28)$ compared to OPLS $(2.52 \AA, \sigma=0.45)$ and CHARMM (2.47 $\AA, \sigma=0.44)$. Interestingly, the apparent improved stability for $\beta$-turns does not seem to be peculiar to a single $\phi, \psi$ combination as there are three class I, four class II, six class III, and one class IV turns (where the class of turn is defined according to $\phi, \psi$ of the middle two residues of the turn $)^{62,63}$, although there may be some restrictions to when this preference may manifest itself, as AMBER did not visit $3_{10}$-like conformations more frequently than CHARMM and OPLS in the IL4 and calbindin simulations. Again, semantic differences make comparison to the discussion of the experimental structures difficult; however, all three simulations have at least one $\mathrm{NH}_{\mathrm{i}} \ldots \mathrm{OC}_{\mathrm{i}-3}$ hydrogen bond in the regions that $3_{10}$-helices were identified in the experimental structures (residues 26-28, 88-91, 123). In addition to an abundance of turns, GPIIA is also proline-rich, having 11 prolines (7\%). Five of these prolines (and two of the three in calbindin) have average $\phi, \psi$ values that are located in the lower left portion of the Ramachandran plot (near $290^{\circ}, 340^{\circ}$ ). Of these residues, the $\psi$ angle in the OPLS simulations is consistently more positive by ca. $20 \ldots$ relative to the CHARMM and AMBER simulations. Inspection of a gas-phase dihedral scan of proline (with $\phi$ unconstrained, but near $290^{\circ}$ ) reveals that a smaller rotational barrier at $\psi=270^{\circ}$ in OPLS leads to a local minimum with a more positive $\psi$ in the same rotational profile using a GB correction for solvation (Fig. 5).

Consideration of whether the local behavior of the peptide bond is consistent between the force fields is necessary for analyzing backbone order parameters, as Buck and Karplus argue that a local order parameter of 0.931 can be attributed to peptide twist $\left(\omega_{1}\right)$ and nitrogen pyramidalization ( $\mathrm{N}-\mathrm{H}$ improper). ${ }^{64}$ The $\omega_{1}$ and $\mathrm{NH}$ improper torsions were averaged over all residues. All three force fields agree that there is a preference for $\omega_{1}<180^{\circ}\left(177-179^{\circ}\right)$, which is in agreement with several surveys of high-resolution crystal structures (average $\omega_{1}$ values reported between 177.0 and 179.6). ${ }^{65-67}$ The differences in these averages are at the limit of their statistical certainty (uncertainty in $\omega_{1}$ is ca. $0.9^{\circ}$ ). The average deviation from planarity is $7-9^{\circ}$, where there is a consistent trend that the deviation from $180^{\circ}$ is in this order: AMBER $>$ OPLS > CHARMM, although again the differences may be insignificant when considering the uncertainty $\left(\mathrm{ca} .0 .5^{\circ}\right.$ ). The rapid fluctuations of the peptide bond about planarity give rise to the difference between the average $\omega_{1}$ and its average displacement from $180^{\circ}$, thus reconciling to some extent why time-averaged J-coupling data is better satisfied by peptide bonds whose values are much closer to $180^{\circ}$ than predicted by ab initio minimizations. ${ }^{68}$ The fact that time-averaged constraints may be more appropriate for NMR refinement has been previously discussed. ${ }^{69}$ The range of the 


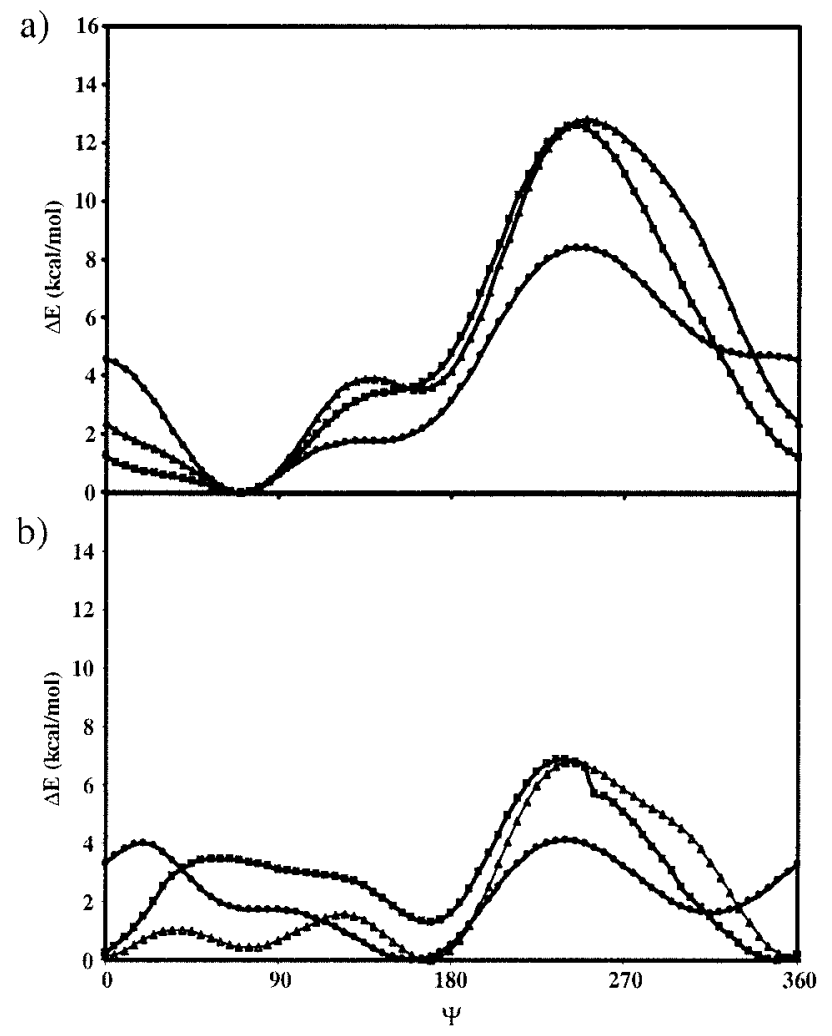

Figure 5. (a) Gas-phase torsional profile for $\psi$ angle of proline with $\phi$ unconstrained but near $290 \ldots$. . (b) The same torsional scan, but with a generalized Born (GB) correction for solvation. The GB calculation was done in each force field's native software to ensure appropriate parameters for the GB equation were used. Symbols are defined according to Figure 4.

average values for the $\mathrm{NH}$ improper angle is also small $\left(1-2^{\circ}\right)$. The $\mathrm{NH}$ improper in the simulations with CHARMM is stiffer than its counterparts (that is, the average displacement from $0^{\circ}$ is slightly smaller). Although OPLS and AMBER may be expected to have similar behavior for this property because they use an identical $\mathrm{V}_{2}$ for the NH improper term, the OPLS simulations show a slight asymmetry in the improper with average values less than $0^{\circ}$. Perhaps most important to probing the effects of local peptide motion on order parameters within the context of the comparison here, the standard deviations for $\omega_{1}$ and the $\mathrm{NH}$ improper are the same to within a degree, suggesting that the mobility of the peptide group is similar among these force fields and will contribute similarly to the backbone order parameters.

\section{Dynamics}

Backbone order parameters $\left(S^{2}\right)$ are the plateau value for the autocorrelation function of the second-order Legendre polynomial of the $\mathrm{N}-\mathrm{H}$ vector, and are frequently derived from NMR relaxation data. As such, they reflect the amount of mobility of the backbone at a given amino acid. The calculated and experimental values are shown for all systems in Figure $6 a-c$. Inspection of the autocorrelation functions themselves shows that regions of low order parameters, particularly loops, have not converged during the simulation. Note that there is an apparent convergence of the order parameter profile as a function of the simulation time (Fig. 6d); however, when comparing the two trajectories of IL4 with CHARMM (Fig. 6b), it is evident that these values are sensitive to the specific trajectory, and therefore conformational changes occurring at a time scale greater than 2 ns. Table 6 lists the average calculated $\mathrm{S}^{2}$ values for the consensus regions of secondary structure, where convergence is more expected. The average experimental $\mathrm{S}^{2}$ for calbindin, IL4 and GPIIA for these regions are 0.85 , 0.90 , and 0.80 respectively. In all cases, there is strong agreement between the force fields and experiment; the former is consistent with the accord between the mobility of $\phi, \psi$ and the peptide bond as indicated by their standard deviations. Qualitatively, the $S^{2}$ values in the loop regions are significantly smaller than experimental measurements. The interpretation of this observation is difficult for two reasons, however. First, the loops may not have been fully equilibrated at the start of the simulation, in which case the trajectory includes the loops transition from the starting geometry to a conformation more preferred by the force field. For example, residues 4,22 , all of loop 3, and some of loop 4 in IL4 all have $S^{2}$ values that increase from 1 to $2 \mathrm{~ns}$ in the first simulation with CHARMM, illustrating that the backbone at those positions may be more rigid than the initial portion of the simulation indicates (Fig. 6d). The fact that $\mathrm{C}_{\alpha}$-RMSD increases in the first $200-500$ ps in most simulations may also suggest that the equilibration of at least part of the protein structure is still occurring at the beginning of the simulation. Second, the calculated order parameters convolute two processes, large time-scale conformational exchange and shorter time-scale fluctuations, whereas large time-scale motions are often ascribed in experiment to a separate exchange term, as described in the context of GPIIA in ref. 29.

Average atomic fluctuations were calculated from structures obtained every 100 time steps ( 0.2 ps); no one force field consistently had the smallest or largest average atomic fluctuations (average values for all atoms and for $\alpha$-carbons involved in secondary structure are listed in Table 6). There is a good correlation between the average atomic fluctuations between simulations of the same protein using the different force fields (correlation coefficient ranges from 0.62 to 0.80 for all atoms), indicating that the same atoms are moving proportionally the same amount in the different simulations. Notably, the $\alpha$-carbons from simulations with AMBER and OPLS were exceptionally well correlated with correlation coefficients between 0.76 and 0.85 . The differences in mobility were not solely localized to the loop regions; the average displacements of the $\alpha$-carbons in the secondary structure alone differed slightly as well (Table 6).

Analysis of side-chain conformational preferences and rate of conformer exchange is hindered by the simulation length. Indeed, only about $15 \%$ of the side chains experience conformational exchange at the $\chi_{1}$ dihedral, and the vast majority of those that do have ca. one to four transitions per simulation (not including proline puckering). The lack of side-chain conformer exchange is not surprising, however; McCammon and Karplus estimated that a rotation of a buried, aromatic side chain occurs on the millisecond time scale. ${ }^{70}$ As a result, the similarity in average $\chi_{1}$ values in Table 5 is most likely an artifact of using identical starting geom- 

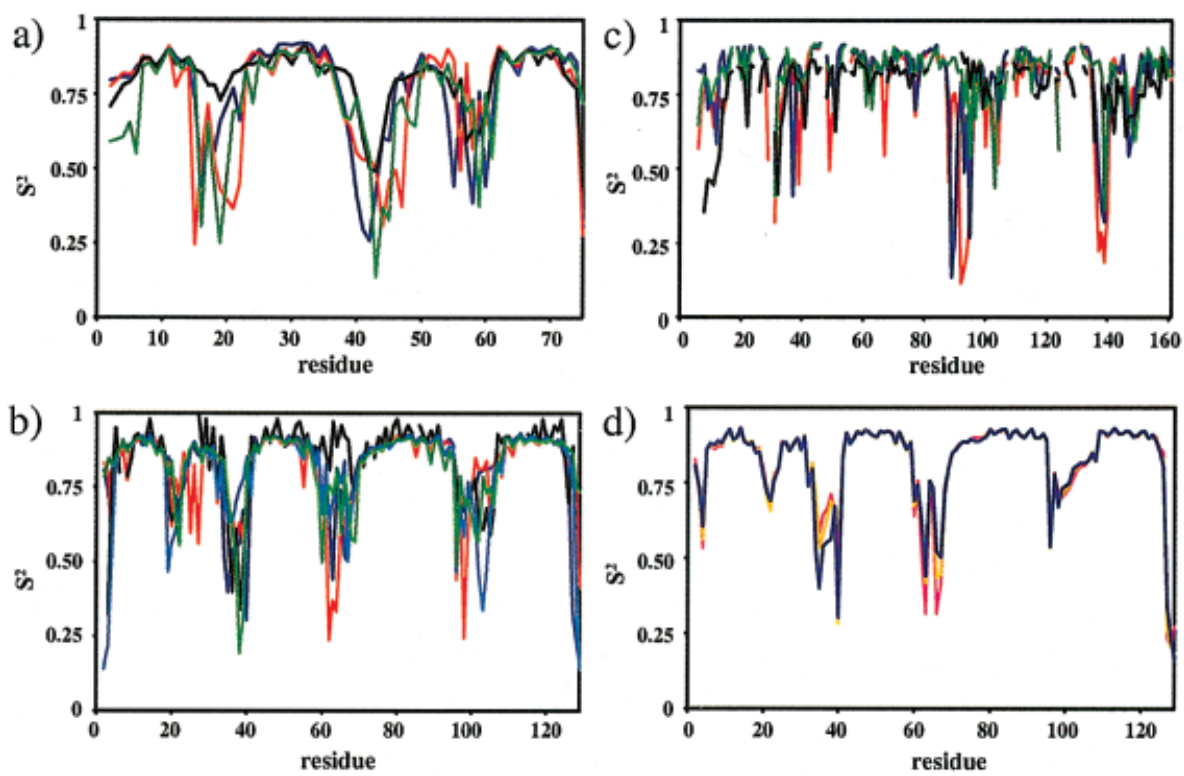

Figure 6. Order parameters for calbindin (a), IL4 (b), GPIIA (c). Colors are defined according to Figure 1; experimental values are shown in black when available. (d) Order parameters for first CHARMM trajectory of IL4 calculated using the first $1 \mathrm{~ns}$ (pink), $1.5 \mathrm{~ns}$ (orange), and full $2 \mathrm{~ns}$ (blue) of the trajectory.

etries, and do not reflect equilibrium conformational preferences. However, there are several trends in side-chain motion here that satisfy intuition. For example, between 62 and $93 \%$ of the residues that undergo conformer exchange at $\chi_{1}$ in all simulations are either glutamine, glutamate, lysine, arginine, serine, or leucine, the first five of which were identified by Zhao and coworkers as particularly mobile residues, based on a comparison of proteins in the PDB that have multiple, independent, experimental structures available. ${ }^{71}$ Likewise, the residues identified by the same study as

Table 6. Dynamic Properties.

\begin{tabular}{llccc}
\hline \multirow{6}{*}{ Calbindin } & & $\begin{array}{c}\text { Atomic } \\
\text { Fluctuations }\end{array}$ & $\begin{array}{c}\text { Atomic Fluctuations } \\
\mathrm{C}_{\alpha}-2^{\circ \mathrm{a}}\end{array}$ \\
& & & & \\
& & & & \\
& AMBER & 0.83 & 1.41 & 0.81 \\
& CHARMM & 0.85 & 1.40 & 0.73 \\
& OPLS & 0.84 & 1.25 & 0.70 \\
& & & & \\
& AMBER & 0.88 & 1.13 & 0.58 \\
\multirow{4}{*}{ GPIIA } & CHARMM 1 & 0.90 & 1.26 & 0.70 \\
& CHARMM 2 & 0.89 & 1.13 & 0.63 \\
& OPLS & 0.88 & 1.08 & 0.53 \\
& AMBER & 0.84 & 0.93 & 0.59 \\
& CHARMM & 0.83 & $1.00^{\mathrm{a}}$ & 0.73 \\
& OPLS & 0.83 & 1.05 & 0.73 \\
\hline
\end{tabular}

asee Table 3 for note on which residues were used for averaging secondary structure properties. less likely to be mobile (isoleucine, threonine, asparagine, aspartate, and the aromatic residues) contribute between 0 and $38 \%$ of these rotations in each simulation. There is no indication that any one force field samples more at the $\chi_{1}$ position overall. Indeed, residues with different $\chi_{1}$ values between force fields were inspected and were found to have, on average, amplified standard deviations in $\chi_{1}$ relative to the bulk average by $15-60 \%$ in all simulations. About $50 \%$ of those residues are charged, and greater than $90 \%$ are solvent exposed. More important to this comparison, the fact that all force fields show enhanced mobility for these residues suggests that these differences are not a result of the torsional exchange occurring exclusively with any one force field.

\section{Discussion and Conclusions}

Each of the force fields tested provided stable protein trajectories throughout several 2-ns simulations, and in no simulation were there significant deviations from the experimental geometry. Furthermore, there was no indication that any of these force fields will consistently sample conformations that are closer to the experimentally determined structures than the others. The location, geometry $\left(\phi, \psi, \omega_{1}\right.$, and $\mathrm{NH}$ improper values and hydrogen-bond lengths) and mobility ( $\mathrm{S}^{2}$ and standard deviations on aforementioned geometrical parameters) of $\alpha$ - and $\beta$-structure were nearly identical, particularly in IL4 and GPIIA. The similarity in $\alpha$ - and $\beta$-geometry and their fluctuations suggests that differences in compactness, as measured by SASA or $r_{\text {gyr }}$, are not a result of any one force field, producing a consistently more compact secondary structure, but instead arises from slightly different tertiary packing 
and loop conformation, and to some extent, side-chain conformation and local folding and refolding events, all of which are expected to occur on time scales longer than $2 \mathrm{~ns}$. The fact that no force field consistently produced the most or least compact structures, that the differences in SASA and $r_{\text {gyr }}$ were similar in size as those between two trajectories with the same force field, and that the average compactness correlated well with the average atomic displacement all suggest that these differences are trajectory-specific, i.e., a result of averaging the quantities over a period in which convergence is not complete. Indeed, differences in the interhelical angles of four independently solved structures of wild-type IL4 indicate potential motion in the tertiary packing. ${ }^{72}$ Amide proton exchange experiments in GPIIA also suggest long time-scale motions (milliseconds to microseconds). ${ }^{29}$ Clearly, longer simulations will be required to model these motions.

In the case of calbindin, the range for average properties between the three force fields is somewhat greater than in the other systems; several properties differed by greater than a standard deviation. However, a consensus picture is still seen between the force fields. Lower order parameters for the secondary structure regions, higher average atomic displacements, longer hydrogenbond lengths, and larger standard deviations on $\phi, \psi$, and hydrogen-bond lengths within $\alpha$ - and $\beta$-structure relative to IL4 for all force fields depict a significantly more mobile protein. Frequent conformational transitions between $\alpha$-helical conformation and

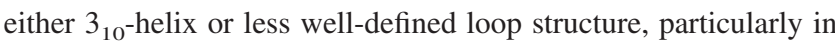
helices 1 and 3, further demonstrates this trend, and is consistent with relatively fast amide proton exchange rates seen for these regions. ${ }^{73}$ The position of helix 3 is poorly defined in the NMR ensemble; the authors of the NMR structure state that sufficient NOEs for high resolution of this region were not obtained because of overlap of leucine side-chain resonances in the homonuclear spectra. ${ }^{21}$ High mobility in this region, which was seen in the simulations with all three force fields, is consistent with poor dispersion and with the relatively low experimental order parameters. In general, helix fraying was suggested as the underlying mechanism for much of the amide proton exchange rate patterns; unfolding of helix termini and stretching motions that may be a precursor to helix fraying was seen on many of the helix ends. In addition to the general mobility of helix ends and the overall mobility of helix 3 , longer than average $i \rightarrow i+4$ hydrogen bonds and transient $i \rightarrow i+3$ backbone interactions at and near residue 13 in all simulations may explain the helical kink noted for that position in the NMR ensemble.

From a cursory glance, the similar performance of these three force fields would seem to disagree with the comparison of several force fields to high-level, quantum mechanical calculations for peptides in the gas phase by Beachy and coworkers, which showed some large discrepancies between the force field's ability to find and rank 10 conformers of alanine tetrapeptide. ${ }^{18}$ However, the authors themselves state that the selected conformations are probably most relevant to denatured proteins, protein loops, and small peptides. In fact, an $\alpha$-helical conformation is not represented in these calculations because it is not a stable conformation for small peptides. As such, this study is complementary to the present one in that the current work emphasizes the geometry and dynamics of secondary structural motifs found in folded proteins ( $\alpha$-helix, $\beta$-sheet, $\gamma$-turn) in solution, but does not address loop structure, as is it difficult to guarantee convergence of loop properties in the time scale of these simulations.

Although this study is certainly not exhaustive in the sense that the examination of a larger set of proteins and force fields is eventually desirable, the force fields studied in the present work are frequently used and the properties monitored are of broad enough scope to clearly classify the gross, subnanosecond behavior of proteins as modeled by these force fields as very similar. Several processes, like side-chain conformer exchange and the transient occupancy of helical conformation, are occasionally observed in the simulations, but require more frequent interchange to construct a well-converged pmf. A more definitive comparison that includes such analyses awaits truly long simulations of a broader range of proteins that will come with advances in hardware akin to that proposed in the Blue Gene Project. ${ }^{74}$

\section{Acknowledgements}

The authors acknowledge Thomas E. Cheatham for the preparation of the AMBER parameter and topology files, William L. Jorgensen for making the BOSS and pepz software packages available, and Michael Crowley for technical assistance and many helpful discussions.

\section{References}

1. Radkiewicz, J. L.; Brooks III, C. L. J Am Chem Soc 2000, 122, 225.

2. Tsui, V.; Case, D. A. J Am Chem Soc 2000, 122, 2489.

3. Plount Price, M. L.; Jorgensen, W. L. J Am Chem Soc 2000, 122, 9455.

4. Duan, Y.; Kollman, P. A. Science 1998, 282, 740.

5. Cornell, W. D.; Cieplak, P.; Bayly, C. I.; Gould, I. R.; Merz, Jr., K. M.; Ferguson, D. M.; Spellmeyer, D. C.; Fox, T.; Caldwell, J. W.; Kollman, P. A. J Am Chem Soc 1995, 117, 5179.

6. Halgren, T. A. J Comp Chem 1996, 17, 490.

7. Jorgensen, W. L.; Maxwell, D. S.; Tirado-Rives, J. J Am Chem Soc 1996, 118, 1125.

8. MacKerell, Jr., A. D.; Bashford, D.; Bellott, M.; Dunbrack, Jr., R. L.; Evanseck, J. D.; Field, M. J.; Fischer, S.; Gao, J.; Guo, H.; Ha, S.; Joseph-McCarthy, D.; Kuchnir, L.; Kuczera, K.; Lau, F. T. K.; Mattos, C.; Michnick, S.; Ngo, T.; Nguyen, D. T.; Prodhom, B.; Reiher, III, W. E.; Roux, B.; Schlenkrich, M.; Smith, J. C.; Stote, R.; Straub, J.; Watanabe, M.; Wirkiewicz-Kuczera, D.; Yin, D.; Karplus, M. J Phys Chem B 1998, 102, 3586.

9. van Gunsteren, W. F.; Billeter, S. R.; Eising, A. A.; Huenenberger, P. H.; Krueger, P.; Mark, A. E.; Scott, W. R. P.; Tironi, I. G. Biomolecular Simulation: The GROMOS96 Manual and User Guide; Hochschulverlag AG/ETH: Zurich, 1996.

10. Price, D. J.; Roberts, J. D.; Jorgensen, W. L. J Am Chem Soc 1998, 120, 9672.

11. Damm, W.; Frontera, A.; Tirado-Rives, J.; Jorgensen, W. L. J Comp Chem 1997, 18, 1955.

12. Rizzo, R. C.; Jorgensen, W. L. J Am Chem Soc 1999, 121, 4827.

13. Hehre, W. J.; Radom, L.; Schleyer, P. V. R.; Pople, J. A. Ab Initio Molecular Orbital Theory; Wiley: New York, 1986.

14. Maxwell, D. S.; Tirado-Rives, J.; Jorgensen, W. L. J Comp Chem 1995, 16, 984.

15. Ajay; Murcko, M. A. J Med Chem 1995, 38, 4953. 
16. Sheinerman, F. B.; Brooks III, C. L. J Mol Biol 1998, 278, 439.

17. Stocker, U.; van Gunsteren, W. F. Proteins Struct Funct Genet 2000, 40, 145.

18. Beachy, M. D.; Chasman, D.; Murphy, R. B.; Halgren, T. A.; Friesner, R. A. J Am Chem Soc 1997, 119, 5908.

19. Head-Gordon, T.; Head-Gordon, M.; Frisch, M. J.; Brooks, III, C. L.; Pople, J. A. J Am Chem Soc 1991, 113, 5989

20. Jorgensen, W. L.; Buckner, J. K. J Phys Chem 1987, 91, 6083.

21. Skelton, N. J.; Kördel, J.; Chazin, W. J. J Mol Biol 1995, 249, 441.

22. Kördel, J.; Skelton, N. J.; Akke, M.; Chazin, W. J. J Mol Biol 1993, 231, 711.

23. Marchand, S.; Roux, B. Proteins Struct Funct Genet 1998, 33, 265.

24. Walter, M. R.; Cook, W. J.; Zhao, B. G.; Cameron, Jr., R. P.; Ealick, S. E.; Walter, Jr., R. L.; Reichert, P.; Naganbushan, T. L.; Trotta, P. P.; Bugg, C. E. J Biol Chem 1992, 267, 20371.

25. Müller, T.; Oehlenschlger, F.; Buehner, M. J Mol Biol 1995, 247, 360.

26. Bonvin, A. J. J.; Brünger, A. J Mol Biol 1995, 250, 80.

27. Akke, M.; Skelton, N. J.; Kördel, J.; Palmer, I. A. G.; Chazin, W. J. Biochemistry 1993, 32, 9832.

28. Redfield, C.; Boyd, J.; Smith, L. J.; Smith, R. A. G.; Dobson, C. M. Biochemistry 1992, 31, 10431.

29. Stone, M. J.; Fairbrother, W. J.; Palmer, I. A. G.; Reizer, J.; Saier, Jr., M. H.; Wright, P. E. Biochemistry 1992, 31, 4394.

30. Goodman, J. L.; Pagel, M. D.; Stone, M. J. J Mol Biol 2000, 295, 963.

31. Brooks, B. R.; Bruccoleri, R. E.; Olafson, B. D.; States, D. J.; Swaminathan, S.; Karplus, M. J Comp Chem 1983, 4, 187.

32. Cheatham, T. E. unpublished work.

33. Jorgensen, W. L. BOSS, version 4.2; Yale University: New Haven, CT, 2000.

34. Case, D. A.; Pearlman, D. A.; Caldwell, J. W.; Cheatham, T. E.; Ross III, T. E.; Simmerling, C. L.; Darden, T.; Merz, Jr., K. M.; Stanton, R. V.; Cheng, A. L.; Vincent, J. J.; Crowley, M.; Fergusson, D. M.; Radmer, R. J.; Seibel, G. L. AMBER6; University of California: San Francisco, CA, 1997.

35. Herzberg, O. J Biol Chem 1992, 267, 24819.

36. Liao, D.; Kapadia, G.; Reddy, P.; Saier, Jr., M. H.; Reizer, J.; Herzberg, O. Biochemistry 1991, 30, 9583.

37. Jorgensen, W. L.; Chandrasehkar, J.; Madura, J. D.; Impey, R. W.; Klein, M. L. J Chem Phys 1983, 79, 926.

38. Reiher, III W. E. thesis (1985).

39. Hockney, R. W. Methods Comput Phys 1970, 9, 136.

40. Potter, D. Computational Physics; Wiley: New York, 1972.

41. Ryckaert, J. P.; Ciccotti, G.; Berendsen, H. J. C. J Comput Phys 1977, $23,327$.

42. Essmann, U.; Perera, L.; Berkowitz, M. L.; Darden, T.; Lee, H.; Pedersen, L. G. J Chem Phys 1995, 103, 8577.

43. Feller, S. E.; Zhang, Y. H.; Pastor, R. W.; Brooks, B. R. J Chem Phys 1995, 103, 4613.

44. Baker, E. N. J Mol Biol 1980, 141, 441.

45. Kabsh, W.; Sander, C. Biopolymers 1983, 22, 2577.

46. Thomas, A.; Bouffioux, O.; Geeurickx, D.; Brasseur, R. Proteins Struct Funct Genet 2001, 43, 28.
47. Allen, M. P.; Tildesley, D. J. Computer Simulations of Liquids; Oxford University Press: Oxford, 1987.

48. Hawkins, G. D.; Cramer, C. J.; Truhlar, D. G. Chem Phys Lett 1995, 246, 122.

49. Still, W. C.; Tempczyk, A.; Hawley, R. C.; Hendrickson, T. J Am Chem Soc 1990, 112, 6127.

50. Dominy, B. N.; Brooks, III, C. L. J Phys Chem B 1999, 103, 3765.

51. Srinivasan, J.; Trevathan, M.; Beroza, P.; Case, D. A. THEOCHEM 1999, 101, 426.

52. IUPAC-IUB Commision on Biochemical Nomenclature. J Mol Biol 1970, 52, 1 .

53. Thompson, P. A.; Eaton, W. A.; Hofrichter, J. Biochemistry 1997, 36, 9200.

54. Kollman, P. A.; Dixon, R.; Cornell, W. D.; Fox, T.; Chipot, C.; Pohoville, A. In Computer Simulations of Biological Systems: Theoretical and Experimental Applications; van Gunsteren, W. F., Weiner, P. K., Wilkinson, A. J., Eds.; Kluwer Academic Publications: Boston, 1997; p 83

55. Soman, K. V.; Karimi, A.; Case, D. A. Biopolymers 1991, 31, 1351.

56. Tirado-Rives, J.; Jorgensen, W. L. Biochemistry 1991, 30, 3864.

57. Young, W. S.; Brooks, III, C. L. J Mol Biol 1996, 259, 560.

58. Daggett, V.; Levitt, M. J Mol Biol 1992, 223, 1121.

59. The result of $\Delta \Delta E=2.4 \mathrm{kcal} / \mathrm{mol}$ was obtained without minimizing the respective monomers.

60. Thomas, A.; Benhabiles, N.; Merisse, R.; Ngwabije, R.; Brasseur, R. Proteins Struct Funct Genet 2001, 43, 37.

61. Chothia, C. Annu Rev Biochem 1984, 53, 537.

62. Lewis, P. N.; Momany, F. A.; Scheraga, H. A. Biochim Biophys Acta 1973, 303, 211

63. Nemethy, G.; Scheraga, H. A. Biochem Biophys Res Commun 1980, $95,320$.

64. Buck, M.; Karplus, M. J Am Chem Soc 1999, 121, 9645.

65. MacArthur, M. W.; Thornton, J. M. J Mol Biol 1996, 264, 1180

66. Marquart, M.; Walter, J.; Deisenhoffer, H.; Bode, W.; Huber, R. Acta Crystallogr B 1983, 39, 480.

67. Sevcik, J.; Dauter, Z.; Lamzin, V. S.; Wilson, K. S. Acta Crystallogr D 1996, 52, 327.

68. Tjandra, N.; Grzesiek, S.; Bax, A. J Am Chem Soc 1996, 118, 6264.

69. Nanzer, A. P.; Poulsen, F. M.; van Gunsteren, W. F.; Torda, A. E. Biochemistry 1994, 33, 14503.

70. McCammon, J. A.; Karplus, M. Biopolymers 1980, 19, 1375.

71. Zhao, S.; Goodsell, D. S.; Olson, A. J. Proteins Struct Funct Genet 2001, 43, 271.

72. Smith, L. J.; Redfield, C.; Smith, R. A. G.; Dobson, C. M.; Clore, G. M.; Gronenborn, A. M.; Walter, M. R.; Naganbushan, T. L.; Wlodawer, A. Nat Struct Biol 1994, 1, 301.

73. Skelton, N. J.; Kördel, J.; Akke, M.; Chazin, W. J. J Mol Biol 1992, 227, 1100 .

74. Swope, W. IBM's Blue Gene Project to Study Protein Folding: Early Status Report, 221st ACS National Meeting: San Diego, CA, 2001

75. Humphrey, W.; Dalke, A.; Schulten, K. J Mol Graphics 1996, 14, 33. 